\title{
Aplicação da análise espacial na avaliação de experimentos de seleção de clones de laranjeira Pêra
}

\author{
Application of the spatial analysis on the evaluation of selection experiments of \\ Pera orange tree clones
}

\section{Emanuel Maia ${ }^{\mathrm{I}}$ Dalmo Lopes de Siqueira ${ }^{\mathrm{II}}$ Sérgio Alves de Carvalho ${ }^{\mathrm{III}}$ Luiz Alexandre Peternelli ${ }^{\mathrm{IV}}$ Rodrigo Rocha Latado ${ }^{\mathrm{II}}$}

\section{RESUMO}

Em experimentos de competição de cultivares de citros, geralmente são utilizados muitos tratamentos, o que requer o emprego de grandes blocos e parcelas com poucas plantas. Tem sido debatido que, nessas condições, pode ocorrer a correlação entre parcelas vizinhas, violando assim a pressuposição de erros independentes da análise de variância. o presente trabalho teve por objetivo avaliar diferentes parametrizações de modelos, considerando ou não a dependência espacial entre parcelas, em dois experimentos de competição de clones de laranjeira Pêra (Citrus sinensis L. Osbeck). Foi utilizada a estrutura auto-regressiva separável de primeira ordem (AR1 x AR1) como modelo de dependência espacial entre os erros. Os resultados encontrados apontam que a modelagem espacial dos erros utilizando modelos autoregressivos separáveis de primeira ordem para experimentos de seleção de clones de laranjeira Pêra, normalmente trazem pequenos ganhos em termos de qualidade de ajuste. A análise desconsiderando o fator blocos mais o ajuste espacial autoregressivo separável de primeira ordem apresentou melhor qualidade de ajuste entre os modelos avaliados.

Palavras-chave: Citrus sinensis, produtividade, volume de copa, autocorrelação espacial, modelos auto-regressivos, melhoramento de plantas.

\section{ABSTRACT}

In competition experiments of citrus cultivations one generally uses many treatments, which requires the use of big blocks and plots with few plants. One has debated that in these conditions there can occur the correlation between neighboring plots, violating, thus, the presupposition of errors independent from the variance analysis. The present work has had as objective to evaluate different model parametrizations, considering or not the spatial dependence between plots, in two competition experiments of Pera orange tree clones (Citrus sinensis L. Osbeck). One has utilized the separable autoregressive structure of first order (AR1 x AR1) as a model of spatial dependence between the errors. The results found indicate that the spatial modeling of the errors by utilizing separable auto-regressive models of first order for selection experiments of Pera orange tree clones normally bring small gains in terms of quality of adjustment. The analysis not considering the block factor plus the separable auto-regressive spatial adjustment of first order has presented better quality of adjustment between the models evaluated.

Key words: Citrus sinensis, productivity, canopy volume, spatial autocorrelation, autoregressive models, plant breeding.

\section{INTRODUÇÃO}

Ensaios de competição de cultivares são de grande valor para os programas de melhoramento de citros, tanto para seleção de cultivares de copa como de porta-enxertos, em várias regiões produtoras de citros

\footnotetext{
'Departamento de Engenharia Florestal, Universidade Federal de Rondônia (UNIR), Av. Norte Sul, 7300, Rolim de Moura, 76940-000, Porto Velho, RO, Brasil. E-mail: emanuel@unir.br. Autor para correspondência.

IIDepartamento de Fitotecnia, Universidade Federal de Viçosa (UFV), Viçosa, MG, Brasil.

IIICentro de Citricultura Sylvio Moreira (IAC), Cordeirópolis, SP, Brasil.

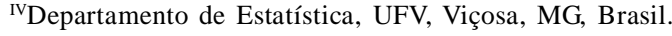


no mundo(DOMINGUES et al., 2003; SMITH et al., 2004; CANTUARIAS-AVILÉS et al., 2010). Nesses estudos, geralmente são empregados muitos tratamentos, com a utilização de grandes áreas experimentais.

Para reduzir os efeitos das variações ambientais sobre os tratamentos, a área é estratificada, com a instalação dos experimentos em blocos casualizados (DOMINGUES et al., 2003; SMITH et al., 2004; CANTUARIAS-AVILÉS et al., 2010), considerando que o controle local e a casualização dos tratamentos são suficientes para garantir uma análise de variância fidedigna. Contudo, vários problemas contribuem para a perda da eficiência neste tipo de análise. Por exemplo, no caso dos citros, a morte de plantas (resultando em perda de parcela) por causa de doenças como Huanglongbing (HLB), clorose variegada dos citros (CVC) e gomose de Phytophthora. Outra questão que pode reduzir a confiabilidade das análises supracitadas é a correlação espacial entre as parcelas (STROUP et al., 1994; SMITH et al., 2001; CASANOVES et al., 2005; RESENDE et al., 2006), pois a análise em blocos casualizados somente é eficiente quando as variações dentro dos blocos são mínimas e as diferenças entre blocos substanciais. A heterogeneidade dentro dos blocos pode resultar em estimativas imprecisas das características dos tratamentos, devido à maior variância associada ao resíduo ou ao favorecimento de algum tratamento (STROUPet al., 1994; DUARTE \& VENCOVSKY, 2005; RESENDEet al., 2006).

O emprego de técnicas capazes de acomodar as variações espaciais, não suportadas pela análise em blocos casualizados, tem sido amplamente utilizado para culturas anuais (SMITH et al., 2001; DUARTE \& VENCOVSKY, 2005; CASANOVES et al., 2005) e, em menor número de trabalhos, para culturas perenes (RESENDE etal., 2006; YE \& JAYAWICKRAMA, 2008). No entanto, até o presente momento, não foram encontradas referências sobre o emprego da abordagem espacial para modelar os erros na análise de variância para experimentos de seleção de cultivares de citros.

Desse modo, o objetivo deste trabalho foi avaliar o desempenho da análise espacial utilizando a estrutura auto-regressiva heterogênea de primeira ordem (AR1 x AR1) em experimentos de seleção de clones de laranjeira Pêra.

\section{MATERIAIS E MÉTODOS}

Os experimentos fazem parte do Programa de melhoramento de Citros do Centro APTA Citros Sylvio Moreira - IAC e são compostos por clones de laranjeira Pêra (Citrus sinensis (L.) Osb.) enxertados sobre o limoeiro Cravo (Citrus limonia Osb.). Para o presente estudo, serão considerados dois experimentos, conduzidos nos municípios de Cordeirópolis e Itapetininga (SP). Estes foram instalados sob o delineamento em blocos completos casualizados, no espaçamento de $7 \times 5 \mathrm{~m}$, com uma planta por parcela.

O primeiro experimento foi instalado no ano de 1997, em Cordeirópolis, contendo sete clones distintos de laranjeira Pêra e seis repetições. O segundo experimento foi instalado no ano de 1998, em Itapetininga, com a avaliação de 20 clones distintos e dez repetições. Foi avaliada produção de frutos em cada planta $(\mathrm{kg}$ planta $^{-1}$ ano $^{-1}$ ), em sete colheitas no experimento de Cordeirópolis e, em cinco colheitas, no de Itapetininga. O volume de copa de cada planta $\left(\mathrm{m}^{3}\right)$ foi avaliada durante três anos, a saber, aos dois, quatro e sete anos após o plantio em Cordeirópolis e aos primeiro, terceiro, quinto anos após o plantio em Itapetininga.

Nas análises, foram considerados três modelos estatísticos: i) em blocos casualizados com erros independentes; ii) em blocos casualizados considerando a dependência espacial auto-regressivo separável de primeira ordem (AR1 x AR1); e iii) desconsiderando a presença dos blocos e adotando somente a análise espacial como controle da variação sistemática. Assim, para os dois primeiros modelos, considerou-se o seguinte modelo generalizado linear misto (e1.)

$$
\mathrm{y}=\mathrm{X} \beta+\mathrm{Z} \tau+\mathrm{e}
$$

em que y é o vetor das observações; $\beta$ é o vetor dos parâmetros fixos de tratamentos; $\tau$ o vetor paramétrico dos efeitos aleatórios de blocos; X e Z são as matrizes de incidência dos efeitos fixos e aleatórios, respectivamente; e e é o vetor dos erros.

A primeira abordagem considera o modelo (e1), delineamento em blocos casualizados - DBC, com análise para erros não correlacionados $\varepsilon \sim \mathrm{NID}\left(0, \mathrm{~s}^{2} \mathrm{I}\right)$. Para o segundo caso, no modelo (e1), foi considerada a correlação espacial dos erros adotando-se a estrutura AR1 x AR1, em que $\varepsilon=\xi+\eta$, na qual $\xi$ representa a variação em pequena escala, modelado como um vetor aleatório com média zero e variâncias espacialmente distribuídas e $\eta$ representa o vetor de erros 
independentes e identicamente distribuídos (CULLIS \& GLEESON, 1991). Para o terceiro modelo, excluiu-se a parametrização $Z \tau$, referente ao controle local, resultando em modelo semelhante ao do delineamento inteiramente casualizado, porém considerando a autocorrelação espacial dos erros, em que $\varepsilon=\xi+\eta$. Todos os modelos foram ajustados utilizando o procedimento da máxima verossimilhança restrita (REML).

Para comparação dos modelos, foi empregado o Critério de Informação de Akaike (AIC) e o teste da razão da máxima verossimilhança. A razão desse teste foi comparada à função densidade de probabilidade $\chi^{2}$ com graus de liberdade compostos pela diferença entre o número de parâmetros da estrutura de covariância de cada modelo (LITTEL et al., 2006). Consideraram-se também as alterações nos valores da amplitude das médias ajustadas para os diferentes modelos e o ordenamento dos clones através da correlação de Spearman.

\section{RESULTADOS E DISCUSSÃO}

De forma geral, os resultados das análises, considerando o ajuste espacial auto-regressivo em duas direções (AR1 x AR1), foram superiores à análise sob erros independentes. Ao verificar os valores para o critério de informação de Akaike (AIC), pode-se observar que há tendência dos modelos serem iguais, com pequenas diferenças nos valores, favorecendo o modelo com dependência espacial, porém sem a parametrização dos blocos (Tabela 1 e Tabela 2). Normalmente, os modelos considerando a dependência espacial entre parcelas adjacentes tendem a ser mais eficientes para as situações em que há maior variância residual (CASANOVES et al., 2005; DUARTE \& VENCOVSKY, 2005). Contudo, nas avaliações deste trabalho, a modelagem espacial da correlação dos erros provocou um aumento nos valores do coeficiente de variação, devido ao aumento da variação residual ou redução nos valores das médias dos tratamentos, visto a redução na amplitude das médias ajustadas (Tabela 1 e Tabela 2). Fato semelhante foi observado em espécies florestais (YE \& JAYAWICKRAMA, 2008).

Observa-se também que os valores encontrados para o coeficiente de autocorrelação encontrados estão normalmente abaixo de 0,7 (Tabela 1 e Tabela 2) e, portando, podem ser considerados baixos quando comparados a outros experimentos nos quais a dependência espacial proporcionou maiores ganhos no ajuste do modelo em relação à análise com erros independentes (RESENDE et al., 2006; YE \& JAYAWICKRAMA, 2008). Esses baixos valores encontrados para a autocorrelação espacial podem estar associados à homogeneidade das áreas experimentais, visto os valores medianos para o coeficiente de variação e a baixa variância para blocos (Tabela 1 e Tabela 2). Desse modo, pode-se considerar que a adição do termo de dependência espacial no modelo usualmente empregado não acarretou em ganhos significativos em termos de ajuste do modelo estatístico.

Os resultados aqui encontrados divergem para as tendências observadas para outras culturas, em que, na maior parte dos casos, a inclusão da autocorrelação espacial acarreta em resultados superiores aos encontrados a partir dos modelos, considerando erros independentes (CASANOVES et al., 2005; RESENDE et al., 2006; YE \& JAYAWICKRAMA, 2008). Entretanto, é importante salientar que, considerando os princípios da estatística experimental, a instalação no delineamento em blocos casualizados, nesses dois experimentos avaliados, pode não ter sido a melhor opção, visto os baixos valores observados para a variância de blocos e a homogeneidade das áreas experimentais.

Vários autores têm demonstrado por estudos via simulação que a técnica do controle local (uso de blocos, especificamente) é o melhor método para controlar gradientes, quando estes são devidamente alocados (FU et al., 1998; SALVADOR et al., 2006). Entretanto, quando esses blocos não forem devidamente alocados, a adoção de modelos que consideram a dependência espacial entre as parcelas adjacentes pode trazer ganhos aos programas de melhoramento genético. Nesse caso, a análise desconsiderando a parametrização dos blocos, associada à dependência espacial dos erros suportou as variações, sendo superior em quatro das sete colheitas realizadas em Cordeirópolis e em duas das cinco colheitas realizadas em Itapetininga. Para a avaliação do volume da copa, esse modelo somente não foi igual ou superior aos demais no terceiro ano em Itapetininga (Tabela 3), indicando ser o melhor procedimento de análise para o caso.

Provavelmente, a homogeneidade da área experimental e os baixos valores para a variância para blocos e para os coeficientes de correlação espacial foram os responsáveis pelas diferenças entre os valores para as médias ajustadas entre os diferentes modelos (Tabela $1 \mathrm{e}$ Tabela 2), causando assim poucas variações no ordenamento dos clones (Tabela 3 ), conforme pode 
Tabela 1. Estimativas do Critério de Informação de Akaike (AIC), coeficiente de variação experimental (CV \%), variância entre blocos ( $\sigma^{2}$ b), coeficiente de autocorrelação residual entre colunas $\left(\rho_{\text {coluna }}\right)$, coeficiente de autocorrelação residual entre linhas $\left(\rho_{\text {linha }}\right)$ e amplitude das médias ajustadas para as análises obtidas no ajuste de modelos no experimento de seleção de clones de laranjeira 'Pêra', conduzido em Cordeirópolis, SP.

\begin{tabular}{|c|c|c|c|c|c|c|}
\hline Modelosi & AIC & $\mathrm{CV}$ & $\sigma_{\mathrm{b}}^{2}$ & $\rho_{\text {coluna }}$ & $\rho_{\text {linha }}$ & Amplitude \\
\hline DBC & 535,00 & 39,61 & 0,00 & & & 10,17 \\
\hline $\mathrm{DBC}+\mathrm{AR}$ & 539,00 & 39,61 & 0,00 & 0,15 & 0,00 & 10,17 \\
\hline $\mathrm{DIC}+\mathrm{AR}$ & 537,00 & 39,61 & & 0,15 & 0,04 & 10,17 \\
\hline DBC & 414,90 & 44,89 & 0,00 & & & 8,25 \\
\hline $\mathrm{DBC}+\mathrm{AR}$ & 410,80 & 44,17 & 0,00 & 0,88 & 0,00 & 9,70 \\
\hline $\mathrm{DIC}+\mathrm{AR}$ & 408,80 & 44,17 & & 0,88 & 0,04 & 9,70 \\
\hline DBC & 565,10 & 44,65 & 0,00 & ano--- & & 13,20 \\
\hline $\mathrm{DBC}+\mathrm{AR}$ & 566,20 & 45,03 & 0,00 & 0,76 & 0,73 & 11,28 \\
\hline $\mathrm{DIC}+\mathrm{AR}$ & 564,20 & 45,03 & & 0,76 & 0,73 & 11,28 \\
\hline DBC & 591,40 & 26,31 & 5,47 & & & 15,67 \\
\hline $\mathrm{DBC}+\mathrm{AR}$ & 591,00 & 26,59 & 0,00 & 0,11 & 0,77 & 15,22 \\
\hline $\mathrm{DIC}+\mathrm{AR}$ & 589,00 & 26,59 & & 0,11 & 0,77 & 15,22 \\
\hline DBC & 434,10 & 12,72 & 0,00 & & & 5,33 \\
\hline $\mathrm{DBC}+\mathrm{AR}$ & 438,10 & 12,72 & 0,00 & 0,49 & 0,00 & 5,34 \\
\hline $\mathrm{DIC}+\mathrm{AR}$ & 436,10 & 12,72 & & 0,49 & 0,04 & 5,34 \\
\hline DBC & 640,90 & 25,66 & 7,55 & ano---. & 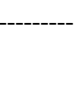 & 52,00 \\
\hline $\mathrm{DBC}+\mathrm{AR}$ & 640,30 & 25,87 & 0,53 & 0,81 & 0,66 & 50,83 \\
\hline $\mathrm{DIC}+\mathrm{AR}$ & 638,30 & 25,89 & & 0,81 & 0,67 & 50,86 \\
\hline DBC & 689,50 & 55,13 & 145,61 & ano-- & & 66,67 \\
\hline $\mathrm{DBC}+\mathrm{AR}$ & 693,00 & 54,92 & 160,45 & 0,74 & 0,00 & 71,26 \\
\hline $\mathrm{DIC}+\mathrm{AR}$ & 694,80 & 58,57 & & 0,16 & 0,43 & 66,48 \\
\hline DBC & $-21,30$ & 41,08 & $\begin{array}{l}\text { lume } d \\
0,00\end{array}$ & após 1 & & 0,23 \\
\hline $\mathrm{DBC}+\mathrm{AR}$ & $-17,50$ & 41,03 & 0,00 & 0,69 & 0,00 & 0,23 \\
\hline $\mathrm{DIC}+\mathrm{AR}$ & $-19,50$ & 41,04 & & 0,69 & 0,22 & 0,23 \\
\hline DBC & 224,80 & 30,08 & $\begin{array}{c}\text { olume d } \\
0,01\end{array}$ & após 1 & & 1,15 \\
\hline $\mathrm{DBC}+\mathrm{AR}$ & 228,80 & 30,10 & 0,01 & 0,07 & 0,00 & 1,15 \\
\hline $\mathrm{DIC}+\mathrm{AR}$ & 226,50 & 30,26 & & 0,08 & 0,60 & 1,19 \\
\hline DBC & 334,30 & 23,72 & $\begin{array}{c}\text { olume d } \\
0,55\end{array}$ & após 1 & & 6,13 \\
\hline $\mathrm{DBC}+\mathrm{AR}$ & 335,50 & 24,18 & 0,40 & 0,78 & 0,72 & 6,60 \\
\hline $\mathrm{DIC}+\mathrm{AR}$ & 334,30 & 25,32 & & 0,78 & 0,77 & 6,36 \\
\hline
\end{tabular}

1 DBC: análise em blocos casualizados; DBC+AR: análise em blocos casualizados mais ajuste espacial auto-regressivo (AR1xAR1); DIC+AR: análise desconsiderando o fator blocos mais ajuste espacial auto-regressivo (AR1xAR1). Células vazias: não se aplica ao modelo. 
Tabela 2. Estimativas do Critério de Informação de Akaike (AIC), coeficiente de variação experimental (CV \%), variância entre blocos ( $\sigma^{2}$ b), coeficiente de autocorrelação residual entre colunas $\left(\rho_{\text {coluna }}\right)$, coeficiente de autocorrelação residual entre linhas $\left(\rho_{\text {linha }}\right)$ e amplitude das médias ajustadas para as análises obtidas no ajuste de modelos no experimento de seleção de clones de laranjeira 'Pêra', conduzido em Itapetininga, SP.

\begin{tabular}{|c|c|c|c|c|c|c|}
\hline M 1 & AIC & $\mathrm{CV}$ & $\sigma_{b}^{2}$ & $\rho_{\text {coluna }}$ & $\rho_{\text {linha }}$ & Amplitude \\
\hline $\mathrm{DBC}$ & 167700 & 3200 & 000 & & & 7105 \\
\hline $\mathrm{DBC}+\mathrm{AR}$ & $1.681,90$ & 32,00 & 0,00 & 0,13 & 0,00 & 71,05 \\
\hline $\mathrm{DIC}+\mathrm{AR}$ & $1.679,90$ & 32,00 & & 0,12 & 0,02 & 71,05 \\
\hline $\mathrm{DBC}$ & $1.830,90$ & 39,94 & 1,51 & & & 63,91 \\
\hline $\mathrm{DBC}+\mathrm{AR}$ & $1.834,90$ & 40,10 & 0,00 & 0,84 & 0,00 & 58,08 \\
\hline $\mathrm{DIC}+\mathrm{AR}$ & $1.832,80$ & 39,82 & & 0,83 & 0,65 & 58,04 \\
\hline DBC & $1.407,40$ & 36,68 & 1,51 & 0,00 & 0,00 & 63,91 \\
\hline $\mathrm{DBC}+\mathrm{AR}$ & $1.405,90$ & 36,72 & 0,00 & 0,84 & 0,00 & 58,08 \\
\hline $\mathrm{DIC}+\mathrm{AR}$ & $1.402,20$ & 37,43 & 0,00 & 0,83 & 0,65 & 58,04 \\
\hline DBC & $1.981,00$ & 36,68 & 128,63 & 0,00 & 0,00 & 171,80 \\
\hline $\mathrm{DBC}+\mathrm{AR}$ & $1.984,90$ & 36,72 & 117,61 & 0,61 & 0,00 & 169,08 \\
\hline $\mathrm{DIC}+\mathrm{AR}$ & $1.981,00$ & 37,43 & 0,00 & 0,63 & 0,68 & 174,39 \\
\hline $\mathrm{DBC}$ & $1.913,00$ & 35,77 & 0,00 & ano---- & & 172,50 \\
\hline $\mathrm{DBC}+\mathrm{AR}$ & $1.914,20$ & 35,79 & 0,00 & 0,65 & 0,66 & 169,66 \\
\hline $\mathrm{DIC}+\mathrm{AR}$ & $1.912,20$ & 35,79 & & 0,65 & 0,66 & 169,66 \\
\hline $\mathrm{DBC}$ & 44,30 & 35,00 & $\begin{array}{l}\text { ume de } \\
0,00\end{array}$ & bapós & & 0,40 \\
\hline $\mathrm{DBC}+\mathrm{AR}$ & 48,30 & 35,00 & 0,00 & 0,12 & 0,00 & 0,40 \\
\hline $\mathrm{DIC}+\mathrm{AR}$ & 46,30 & 35,00 & & 0,11 & 0,03 & 0,40 \\
\hline DBC & 596,50 & 30,72 & 0,02 & & & 1,02 \\
\hline $\mathrm{DBC}+\mathrm{AR}$ & 583,30 & 30,53 & 0,03 & 0,85 & 0,00 & 0,92 \\
\hline $\mathrm{DIC}+\mathrm{AR}$ & 582,30 & 30,90 & & 0,85 & 0,05 & 0,93 \\
\hline DBC & $1.012,70$ & 26,79 & $\begin{array}{l}\text { ume de } \\
0,79\end{array}$ & após o & & 16,42 \\
\hline $\mathrm{DBC}+\mathrm{AR}$ & $1.016,60$ & 26,79 & 0,80 & 0,60 & 0,00 & 16,36 \\
\hline $\mathrm{DIC}+\mathrm{AR}$ & $1.017,90$ & 27,60 & & 0,48 & 0,54 & 16,52 \\
\hline
\end{tabular}

${ }^{1}$ DBC: análise em blocos casualizados; DBC+AR: análise em blocos casualizados mais ajuste espacial auto-regressivo (AR1xAR1); DIC+AR: análise desconsiderando o fator blocos mais ajuste espacial auto-regressivo (AR1xAR1). Células vazias: não se aplica ao modelo.

ser observado a partir dos valores do coeficiente da correlação de Spearman.

\section{CONCLUSÃO}

A modelagem espacial dos erros utilizando modelos auto-regressivos separáveis de primeira ordem, para experimentos de seleção de clones de laranjeira Pêra, normalmente trazem ganhos pequenos, porém significativos. A análise desconsiderando o fator blocos e usando apenas o ajuste espacial autoregressivo separável de primeira ordem apresentou melhor qualidade de ajuste entre os modelos avaliados. 
Tabela 3. Valores de probabilidade associados ao teste da razão da máxima verossimilhança (p-LTR) e coeficiente de correlação de Spearman $(\rho)$ entre diferentes modelos para experimentos de seleção de clones de laranjeira 'Pêra'.

\begin{tabular}{|c|c|c|c|c|}
\hline \multirow{3}{*}{ Comparações } & & ópolis- & \multirow[b]{2}{*}{ p-LTR } & \multirow[t]{2}{*}{ ininga } \\
\hline & $\mathrm{p}$-LTR & $\rho$ & & \\
\hline & \multicolumn{2}{|c|}{ 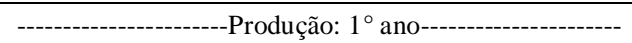 } & \multicolumn{2}{|c|}{----------------------Produção: $1^{\circ}$ ano----------------------- } \\
\hline $\mathrm{DBC} \times \mathrm{DBC}+\mathrm{AR}$ & 1,000 & 0,9989 & 1,000 & 1,0000 \\
\hline $\mathrm{DBC} \times \mathrm{DIC}+\mathrm{AR}$ & 1,000 & 0,9989 & 1,000 & 1,0000 \\
\hline \multirow[t]{2}{*}{$\mathrm{DIC}+\mathrm{AR} \times \mathrm{DBC}+\mathrm{AR}$} & 1,000 & 1,0000 & 1,000 & 1,0000 \\
\hline & \multicolumn{2}{|c|}{ 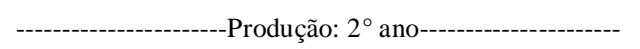 } & \multicolumn{2}{|c|}{--Produção: $2^{\circ}$ ano------ } \\
\hline $\mathrm{DBC} \times \mathrm{DBC}+\mathrm{AR}$ & 0,017 & 0,9516 & 1,000 & 1,0000 \\
\hline $\mathrm{DBC} \times \mathrm{DIC}+\mathrm{AR}$ & 0,004 & 0,9516 & 0,752 & 1,0000 \\
\hline \multirow[t]{2}{*}{$\mathrm{DIC}+\mathrm{AR} \times \mathrm{DBC}+\mathrm{AR}$} & 1,000 & 1,0000 & 0,752 & 1,0000 \\
\hline & \multicolumn{2}{|c|}{ - } & \multicolumn{2}{|c|}{----'Produção: $3^{\circ}$ ano----- } \\
\hline $\mathrm{DBC} \times \mathrm{DBC}+\mathrm{AR}$ & 0,235 & 0,9405 & 0,064 & 0,9865 \\
\hline $\mathrm{DBC} \times \mathrm{DIC}+\mathrm{AR}$ & 0,089 & 0,9405 & 0,007 & 0,9850 \\
\hline \multirow[t]{2}{*}{$\mathrm{DIC}+\mathrm{AR} \times \mathrm{DBC}+\mathrm{AR}$} & 1,000 & 1,0000 & 0,192 & 0,9955 \\
\hline & \multicolumn{2}{|c|}{ 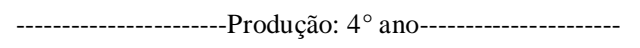 } & \multicolumn{2}{|c|}{---'Produção: $4^{\circ}$ ano----- } \\
\hline $\mathrm{DBC} \times \mathrm{DBC}+\mathrm{AR}$ & 0,111 & 0,9517 & 0,951 & 0,9895 \\
\hline $\mathrm{DBC} \times \mathrm{DIC}+\mathrm{AR}$ & 0,036 & 0,9517 & 0,157 & 0,9985 \\
\hline \multirow[t]{2}{*}{$\mathrm{DIC}+\mathrm{AR} \times \mathrm{DBC}+\mathrm{AR}$} & 1,000 & 1,0000 & 0,168 & 0,9910 \\
\hline & \multicolumn{2}{|c|}{ 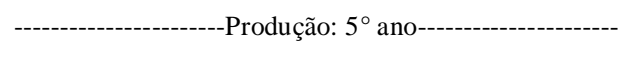 } & \multicolumn{2}{|c|}{ 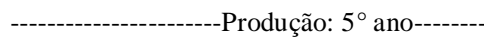 } \\
\hline $\mathrm{DBC} \times \mathrm{DBC}+\mathrm{AR}$ & 1,000 & 0,9945 & 0,247 & 0,9835 \\
\hline $\mathrm{DBC} \times \mathrm{DIC}+\mathrm{AR}$ & 1,000 & 0,9945 & 0,094 & 0,9835 \\
\hline \multirow[t]{2}{*}{$\mathrm{DIC}+\mathrm{AR} \times \mathrm{DBC}+\mathrm{AR}$} & 1,000 & 1,0000 & 1,000 & 1,0000 \\
\hline & \multicolumn{2}{|c|}{ 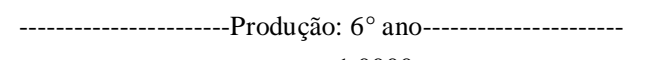 } & \multicolumn{2}{|c|}{-------------Volume de copa: $1^{\circ}$ ano------------- } \\
\hline $\mathrm{DBC} \times \mathrm{DBC}+\mathrm{AR}$ & 0,100 & 1,0000 & 1,000 & 1,0000 \\
\hline $\mathrm{DBC} \times \mathrm{DIC}+\mathrm{AR}$ & 0,032 & 1,0000 & 1,000 & 1,0000 \\
\hline \multirow[t]{2}{*}{$\mathrm{DIC}+\mathrm{AR} \times \mathrm{DBC}+\mathrm{AR}$} & 1,000 & 1,0000 & 1,000 & 1,0000 \\
\hline & \multicolumn{2}{|c|}{ 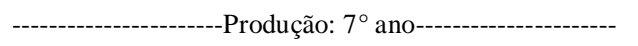 } & \multicolumn{2}{|c|}{ - Volume de copa: $3^{\circ}$ ano--- } \\
\hline $\mathrm{DBC} \times \mathrm{DBC}+\mathrm{AR}$ & 0,779 & 0,9604 & $<0,001$ & 1,0000 \\
\hline $\mathrm{DBC} \times \mathrm{DIC}+\mathrm{AR}$ & 0,069 & 0,9824 & $<0,001$ & 0,8902 \\
\hline \multirow[t]{2}{*}{$\mathrm{DIC}+\mathrm{AR} \times \mathrm{DBC}+\mathrm{AR}$} & 0,051 & 0,9692 & 0,317 & 0,8902 \\
\hline & \multicolumn{2}{|c|}{-------------Volume de copa: $2^{\circ}$ ano------------ } & ------------------V & opa: $5^{\circ}$ ano----- \\
\hline $\mathrm{DBC} \times \mathrm{DBC}+\mathrm{AR}$ & 0,905 & 0,9912 & 0,951 & 0,9970 \\
\hline $\mathrm{DBC} \times \mathrm{DIC}+\mathrm{AR}$ & 0,655 & 0,9912 & 0,074 & 0,9955 \\
\hline $\mathrm{DIC}+\mathrm{AR} \times \mathrm{DBC}+\mathrm{AR}$ & 1,000 & 1,0000 & 0,069 & 0,9955 \\
\hline & ----------------Vo & copa: $4^{\circ}$ ano---- & & \\
\hline $\mathrm{DBC} \times \mathrm{DBC}+\mathrm{AR}$ & 1,000 & 0,9956 & & \\
\hline $\mathrm{DBC} \times \mathrm{DIC}+\mathrm{AR}$ & 0,584 & 0,9956 & & \\
\hline $\mathrm{DIC}+\mathrm{AR} \times \mathrm{DBC}+\mathrm{AR}$ & 0,584 & 1,0000 & & \\
\hline & -----------------Vo & opa: $7^{\circ}$ ano----- & & \\
\hline $\mathrm{DBC} \times \mathrm{DBC}+\mathrm{AR}$ & 0,247 & 0,9956 & & \\
\hline $\mathrm{DBC} \times \mathrm{DIC}+\mathrm{AR}$ & 0,157 & 0,9956 & & \\
\hline $\mathrm{DIC}+\mathrm{AR} \times \mathrm{DBC}+\mathrm{AR}$ & 0,371 & 1,0000 & & \\
\hline
\end{tabular}

${ }^{1}$ DBC: análise em blocos casualizados; DBC+AR: análise em blocos casualizados mais ajuste espacial auto-regressivo (AR1xAR1); DIC+AR: análise desconsiderando o fator blocos mais ajuste espacial auto-regressivo (AR1xAR1). Células vazias: não se aplica. 


\section{AGRADECIMENTOS}

Agradecemos ao Conselho Nacional de Desenvolvimento Científico e Tecnológico (CNPq) pelo apoio financeiro na condução dos experimentos de campo (Processo 485839/ 2006-7) e concessão de bolsa de doutorado ao primeiro autor.

\section{REFERÊNCIAS}

CANTUARIAS-AVILÉS, T. et al. Tree performance and fruit yield and quality of 'Okitsu' Satsuma mandarin grafted on 12 rootstocks. Scientia Horticulturae, v.123, p. 318-322, 2010. Disponível em: 〈http://dx.doi.org/10.1016/j.scienta.2009.09.020〉. Acesso em: 11 jan. 2010. doi: 10.1016/j.scienta.2009.09.020.

CASANOVES, F. et al. Error Variation in multienvironment peanut trials: Within-trial spatial correlation and between-trial heterogeneity. Crop Science, v.45, p.1927-1933, 2005. Disponível em: <https://www.crops.org/publications/cs/abstracts/ 45/5/1927>. Acesso em: 12 out. 2009. doi: 10.2135/ cropsci2004.0547.

CULLIS, B.R.; GLEESON, A.C. Spatial analysis of field experiments-an extension to two dimentions. Biometrics, v.47, p.1449-1460, 1991. Disponível em: <http://www.jstor.org/ stable/2532398>. Acesso em: 12 out. 2009.

DOMINGUES, E.T. et al. Seleção de variedades de laranja quanto à qualidade do fruto e período de maturação. Laranja, v.24, p.471-490, 2003. Disponível em: <http://revistalaranja.centrodecitricultura.br/ index.php?pag=edicoes_revista\&edicaon=11>. Acesso em: 02 mar. 2009.

DUARTE, J.B.; VENCOVSKY, R. Spatial statistical analysis and selection of genotypes in plant breeding. Pesquisa Agropecúaria Brasileira, v.40, p.107-114, 2005. Disponível em: <http:// dx.doi.org/10.1590/S0100-204X2005000200002>. Acesso em: 08 out. 2009. doi: 10.1590/S0100-204X2005000200002.

FU Y.B. et al. Incomplete block designs for genetic testing: statistical efficiencies of estimating family means. Canadian Journal of Forest Research, v.28, p.977-986, 1998. Disponível em: <http://www.nrcresearchpress.com/doi/abs/ 10.1139/x98-072>. Acesso em: 11 jan. 2010. doi: 10.1139/ x98-072.

LITTELL, R.C. et al. SAS for Mixed Models. $2^{\text {a. }}$ ed. Cary: SAS Institute Inc., 2006. 831p.

RESENDE, M.D.V. et al. Multivariate spatial statistical analysis of longitudinal data in perennial crops. Revista de Matemática e Estatística, v.24, p.147-169, 2006. Disponível em: <http:/ /jaguar.fcav.unesp.br/RME/fasciculos/v24/v24_n1/ indice_v24_n1.php>. Acesso em: 07 jan. 2010.

SALVADOR, A.G. et al. Comparison of experimental designs for clonal forestry using simulated data. Forest Science, v.52, p.108116, 2006. Disponível em: <http://www.ingentaconnect.com/ content/saf/fs/2006/00000052/00000001/art00010>. Acesso em: 05 fev. 2010.

SMITH, A. et al.. Analyzing variety by environment data using multiplicative mixed models and adjustments for spatial field trend. Biometrics, v.57, p.1138-1147, 2001. Disponível em: <http:// onlinelibrary.wiley.com/doi/10.1111/j.0006-341X.2001.01138.x/ pdf $>$. Acesso em: 05 fev. 2010. doi: 10.1111/j.0006341X.2001.01138.x.

SMITH, M.W. et al. Long-term performance of 'Ellendale' mandarin on seven commercial rootstocks in sub-tropical Australia. Scientia Horticulturae, v.102, p.75-89, 2004. Disponível em: <http://dx.doi.org/10.1016/j.scienta.2003.12.004>. Acesso em: 12 out. 2010. doi: 10.1016/j.scienta.2003.12.004.

STROUP, W.W. et al. Removing Spatial Variation from Wheat Yield Trials: A Comparison of Methods. Crop Science, v.34, p.62-66, 1994. Disponível em: <https://www.crops.org/ publications/cs/abstracts/34/1/CS0340010062 >. Acesso em: 02 fev. 2010. doi: 10.2135/cropsci1994.0011183X003400010011x.

YE, T.Z.; JAYAWICKRAMA, K.J.S. Efficiency of using spatial analysis in first-generation coastal Douglas-fir progeny tests in the US Pacific Northwest. Tree Genetics \& Genomes, v.4, p.677-692, 2008. Disponível em: <http:// www.springerlink.com/content/453 w $757 \mathrm{~m} 2621$ w5 $73 /$ ?MUD=MP >. Acesso em: 12 out. 2010. doi: 10.1007/s11295008-0142-4. 